\title{
3-D and Power Doppler Ultrasound in Translational Research
}

\author{
${ }^{1}$ Lakshmanaswamy Rajkumar, ${ }^{2}$ Sanja Plavsic Kupesic
}

${ }^{1}$ Basic Science Research Director, Center of Excellence in Cancer Research, Department of Biomedical Sciences, Paul L Foster School of Medicine, Texas Tech University Health Sciences Center, 5001 El Paso Drive, El Paso, Texas 79905, USA

${ }^{2}$ Professor and Clinical Professor of Obstetrics and Gynecology and Radiology, Department of Medical Education, Paul L Foster School of Medicine, Texas Tech University Health Sciences Center, 5001 El Paso Drive, EI Paso, Texas 79905, USA

Correspondence: Lakshmanaswamy Rajkumar, Basic Science Research Director, Center of Excellence in Cancer Research Department of Biomedical Sciences, Paul L Foster School of Medicine, Texas Tech University Health Sciences Center, 5001 El Paso Drive, El Paso, Texas 79905, USA, Phone: (915) 783-5235, Fax: (915) 783-5222

e-mail: rajkumar.lakshmanaswamy@ttuhsc.edu

\begin{abstract}
At Texas Tech University Health Sciences Center in El Paso we have developed an animal model to study mammary carcinogenesis and neovascularization. Seven-week-old female Sprague Dawley rats are injected with N-methyl-N-nitrosourea (MNU) intraperitoneally at a concentration of $50 \mathrm{mg} / \mathrm{kg}$ bodyweight. The rats are palpated twice a week for development of mammary gland tumors. On the appearance of the first palpable tumor, the animals are divided into the groups treated with aromatase inhibitor, ethanolic neem leaf extract and control group, receiving vehicle treatment. Using a high-resolution $16 \mathrm{MHz}$ linear array transducer mammary glands are imaged before and every two weeks following the introduction of the therapy. 3-D US coupled with 3-D power Doppler imaging is used for evaluation of the volume and vascularity of the mammary gland lesions. Pulsatility and resistance index and vascularity, flow and vascularity flow indices are evaluated longitudinally. Our preliminary data indicate that 2-D and 3-D power Doppler ultrasound may be efficiently used for evaluation of the mammary gland tumor volumes and vascularity. 2-D and 3-D power Doppler ultrasound may also be used for evaluation of the response of mammary gland tumors to different lines of medication therapy.
\end{abstract}

Keywords: Mammary gland tumors, animal models, 3-D ultrasound imaging, color Doppler imaging, 3-D power Doppler imaging.

\section{INTRODUCTION}

At Texas Tech University Health Sciences Center in El Paso we have established two animal model systems (rats and mice) to study mammary carcinogenesis and neovascularization. A typical rat model experiment for hormonedependent mammary carcinogenesis involves the treatment of virgin animals or ovariectomized animals with hormones, antihormones, plant products, drugs or growth factors followed by carcinogen injection. In alternative scenario, the carcinogen is injected before the treatment with hormones, antihormones, plant products, growth factors or antiangiogenetic drugs. Transgenic mice over expressing HER-2/neu gene are used to study the same in a hormoneindependent system. These two models account for the majority of the types of breast cancer observed in humans. Furthermore, the mammary tumors induced by administration of chemical carcinogen are morphologically and histologically similar to human breast cancer. ${ }^{1}$

\section{ULTRASOUND AND COLOR DOPPLER STUDIES OF MAMMARY GLAND TUMORS IN RAT MODELS}

Seven weeks old female Sprague Dawley rats are injected with $\mathrm{N}$-methyl-N-nitrosourea (MNU) intraperitoneally at a concentration of $50 \mathrm{mg} / \mathrm{kg}$ bodyweight. The rats are palpated twice a week for development of mammary gland tumors. On the appearance of the first palpable tumor, the animals are divided into the following groups: (1) Aromatase inhibitor treated, (2) Ethanolic neem leaf extract treated, and (3) Control group, receiving vehicle treatment. All treatments are given for four weeks by daily intraperitoneal injections.

Mammary cancers in rats are located just underneath the skin, and high frequency ultrasound could be used for their visualization (Fig. 1). Three-dimensional (3-D) and color/power Doppler ultrasound is safe, noninvasive, easy to use, and provide important diagnostic information. Sonographic assessment is capable of revealing the 


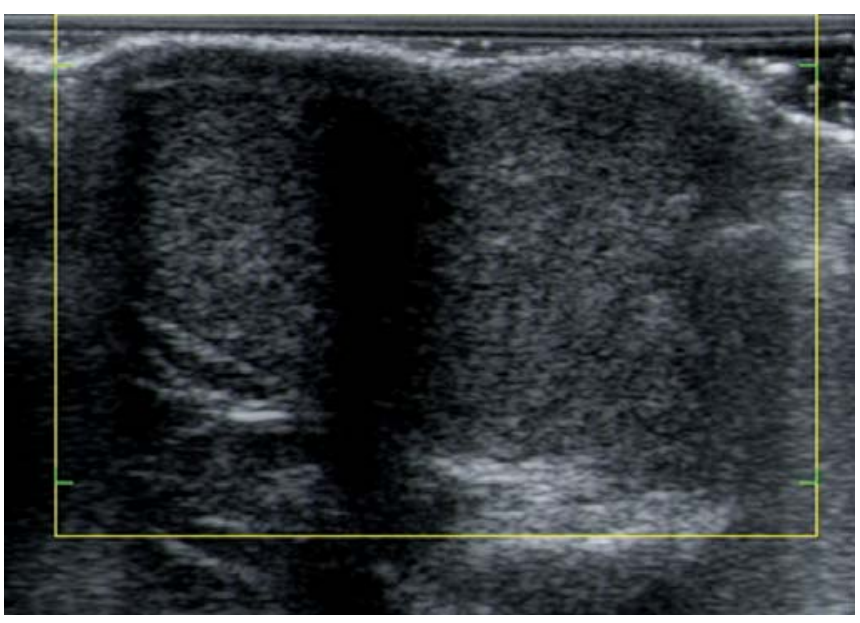

Fig. 1: 2-D ultrasound of a rat mammary gland tumor

3-dimensional size and volume of the mammary gland tumor, as well as outlining the subcutaneous lesions and assessing their relation to adjacent structures. Moreover, information about the lesion quality (solid, cystic, and combined) and the inner structure (homogeneous, inhomogeneous, hypoechoic, hyperechoic, calcification foci, and necroses) can be obtained. Power Doppler ultrasound may be used to qualitatively analyze the distribution and branching pattern of the newly formed vessels (Fig. 2), while pulsed Doppler wave form analysis provides information on blood flow impedance in mammary gland tumor vessels (Fig. 3).

In our study mammary gland cancers are imaged with a high-resolution $16 \mathrm{MHz}$ linear array transducer using a Voluson i8 machine (GE Medical Systems, Milwaukee, USA). The lesion quality (solid, cystic or complex) and echotexture (homogeneous/inhomogeneous, hypoechoic/ hyperechoic, presence/absence of calcification foci) is defined.

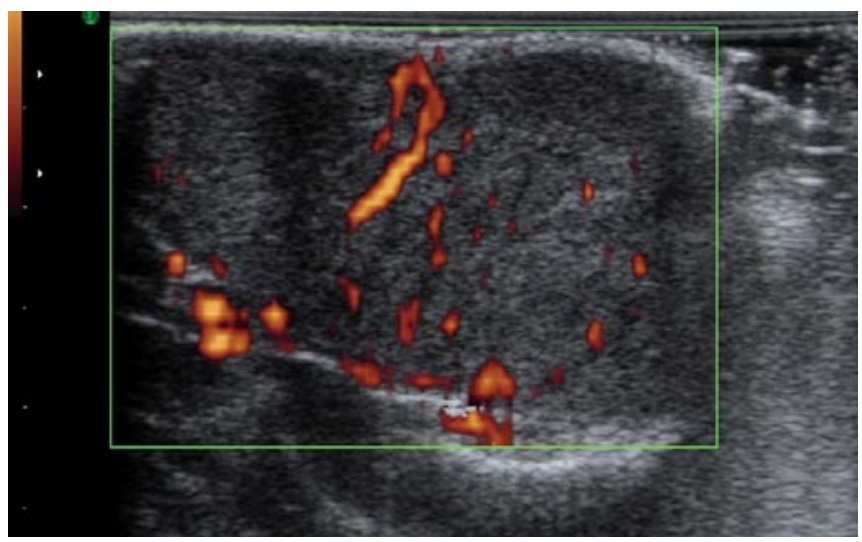

Fig. 2: 2-D color Doppler of mammary gland neovascularization. Note the penetrating pattern of newly formed vessels

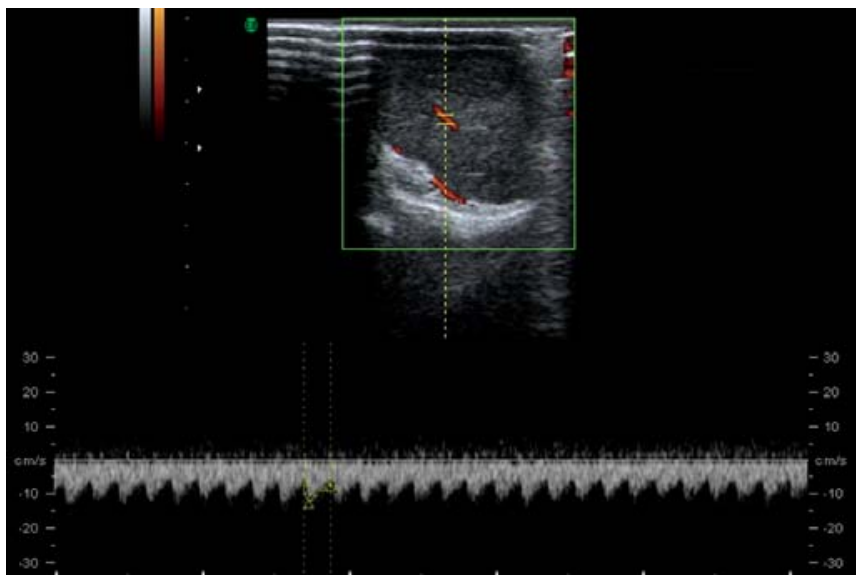

Fig. 3: Pulsed Doppler waveform analysis. Low vascular impedance blood flow signals (pulsatility index of 0.52 ) are typical for neovascularization

Ultrasound measurements of a mean maximum anteroposterior and transverse diameter of mammary gland tumors are expressed prior to introduction of the therapy, and compared to the same diameters post-therapy.

Following B-mode assessment each mammary gland tumor is evaluated by 2-D color and 3-D power Doppler ultrasound. Microcirculation is qualitatively (distribution of the vessels and branching pattern assessment) and quantitatively evaluated. The equipment was set to achieve the maximum sensitivity so as to detect the low-velocity flow with least noise (power Doppler gain-high (90-94\%), wall filters-low, pulsed repetition frequency-0.9 kHz). Pulsed Doppler waveform analysis is performed to assess resistance (S-D/S) and pulsatility index (S-D/mean) for each mammary gland tumor (Fig. 3).

3-D US coupled with 3-D power Doppler imaging are used for evaluation of the volume and vascularity of the mammary gland tumors (Figs 4 and 5). Three parameters are analyzed in our prospective studies. The vascularization index (VI), which expresses as a percentage of the number of color $v s$ grey voxels in the given volume, and represents the number of the vessels within the studied tissue volume. The flow index (FI), which measures the intensity of blood flow and will be calculated automatically by the built-in computer: FI = weighted color values/color values. The vascular flow index (VFI), which represents the average color value of all grey and color voxels, and expresses both the blood flow and vascularization.

2-D, 2-D color Doppler (pulsatility and resistance index) and 3-D power Doppler ultrasound (vascularity, flow and vascularity flow indices) are analyzed before, and every 


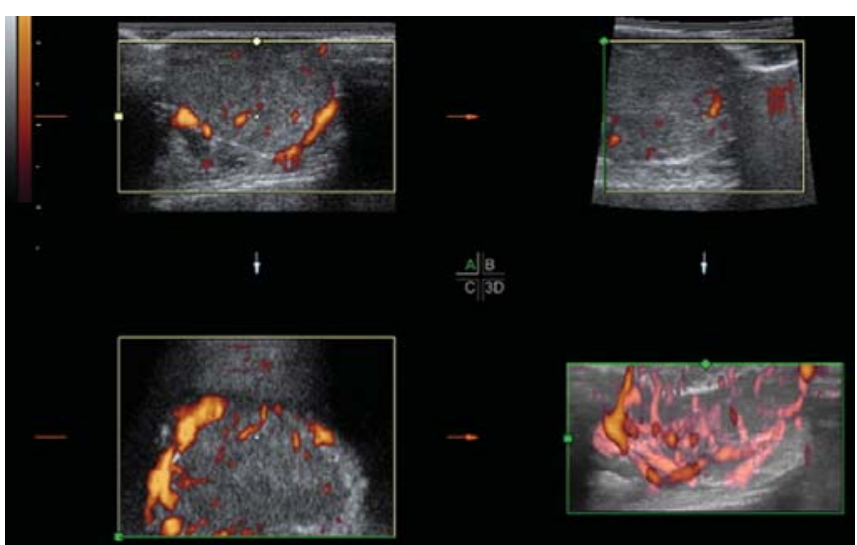

Fig. 4: 3-D power Doppler and gray scale ultrasound of mammary gland tumor neovascularization. Note irregular course and penetrating pattern of the vessels

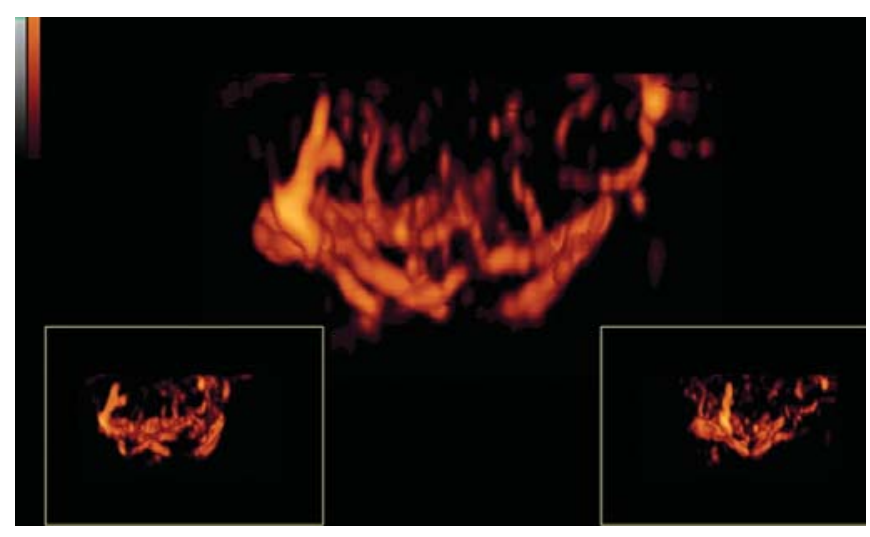

Fig. 5: 3-D power Doppler image of mammary gland tumor neovascularization in a rat model

two weeks following the introduction of the therapy. The efficacy of treatment is evaluated by looking at the volumetric increase or reduction of the tumor, and changes in the quality of the vascular signals.

Ultrasound results are compared to the specimens obtained either by surgery or biopsy.

\section{CANCER RESEARCH AND ULTRASOUND}

The main advantages of ultrasound are lack of ionizing irradiation, low cost, opportunity to perform longitudinal studies, and noninvasiveness. Cancer research is one of the major areas being largely impacted by ultrasonography. We find that translational research using ultrasound imaging is very useful for teaching our students about basic concepts of cancerogenesis, angiogenesis and neovascularization.

Ultrasound imaging complement the assessments based on pathology, genomics, and proteomics. ${ }^{2}$ Using the state- of-the-art ultrasound imaging technology in cancer research one can detect noninvasively specific molecular changes associated with the presence of early lesions and malignant disease. Ultrasound modality is helpful in identifying disease recurrence and response to various therapies.

In preclinical research ultrasound imaging provides a rather powerful tool for various aspects of drug development. Animal models of human tumors are regularly used by researchers to test the efficacy of different lines of therapy. Ultrasound imaging of animal models allows drug target validation and monitoring of therapeutic efficacy in tumors with different combinations of genetic alterations. ${ }^{3,4}$ Data obtained from animal models can be efficiently and effectively used for in vivo molecular targeted imaging in cancer patients.

In vivo ultrasound imaging offers a great potential in the preclinical testing phase to determine a product's ultimate safety profile and toxicity.

As demonstrated in our images, 2-D and 3-D color and power Doppler ultrasound is useful in early visualization and follow-up of tumor neovascularization. Understanding the process of angiogenesis during cancer progression is extremely important. If antiangiogenic drugs are developed to treat breast cancer, ultrasound may become a method of choice for longitudinal and noninvasive follow-up of the antiangiogenic effect of these medications, which is of imminence importance to accelerate drug research and development.

Basic carcinogenesis experiments measure tumor volume and growth rates using crude measurements by calipers. Allowing precise and objective volume assessment, 3-D ultrasound truly gives another dimension to this type of research. Ultrasound has a potential to determine the significance of neovascularization for tumor progression, based on evaluation of the process and intensity of angiogenesis from the day of carcinogenic insult until the formation of the palpable tumor.

Using ultrasound guided procedures researchers may study the effect of locally introduced medications. Their effect can be evaluated longitudinally, without the need of performing survival animal surgeries.

\section{CONCLUSION}

Our preliminary data indicate that 2-D and 3-D power Doppler ultrasound may be efficiently used for evaluation of the mammary gland tumor volumes and vascularity. 
2-D and 3-D power Doppler ultrasound may also be used for evaluation of the response of mammary gland tumors to different lines of medication therapy.

\section{REFERENCES}

1. Malejka-Giganti D, Bennett KK, Culp SJ, Beland FA, Shinozuka $\mathrm{H}$, et al. Suppression of 7,12-dimethylbenz (a) anthraceneinduced mammary carcinogenesis by preinitiation treatment of rats with beta-naphthoflavone coincides with decreased levels of the carcinogen-derived DNA adducts in the mammary gland. Cancer Detect Prev 2005;29(4):338-47.
2. Misek DE, Imafuku Y, Hanash SM. Application of proteomic technologies to tumor analysis. Pharmacogenomics 2004;5(8):1129-37.

3. Wang S, El-Deiry WS. Inducible silencing of KILLER/DR5 in vivo promotes bioluminescent colon tumor xenograft growth and confers resistance to chemotherapeutic agent 5-fluorouracil. Cancer Res Sep 2004;15;64(18):6666-72.

4. Wang W, El-Deiry WS. Bioluminescent molecular imaging of endogenous and exogenous p53-mediated transcription in vitro and in vivo using an HCT116 human colon carcinoma xenograft model. Cancer Biol Ther 2003;2(2):196-202. 